\title{
Radio observations of the circumnuclear ring in NGC 6951
}

\author{
D. J. Saikia ${ }^{1,2,3}$, B. Phookun ${ }^{4}$, A. Pedlar ${ }^{2,5}$, and K. Kohno 6,7 \\ 1 Department of Physics, Queen's University, Kingston, Ontario, K7L 3N6, Canada \\ 2 Jodrell Bank Observatory, University of Manchester, Macclesfield, Cheshire SK11 9DL, UK \\ 3 National Centre for Radio Astrophysics, Tata Institute of Fundamental Research, Post Bag No. 3, \\ Ganeshkhind, Pune 411 007, India \\ 4 St. Stephen's College, University of Delhi, Delhi 110 007, India \\ 5 Onsala Space Observatory, 43992 Onsala, Sweden \\ 6 Nobeyama Radio Observatory, Minamimaki, Minamisaku, Nagano 384-1305, Japan \\ 7 Institute of Astronomy, University of Tokyo, Osawa, Mitaka, Tokyo 181-0015, Japan
}

Received 26 September 2001 / Accepted 3 December 2001

\begin{abstract}
We present sensitive, high-resolution radio observations of the circumnuclear region of the barred spiral galaxy NGC6951. These observations reveal a ring of radio emission with many discrete components and a marginally resolved nuclear component. We compare the radio ring with observations at other wavelengths, and discuss the nature of the compact radio components.
\end{abstract}

Key words. galaxies: active - galaxies: individual: NGC6951 - galaxies: nuclei - galaxies: spiral - radio continuum: galaxies

\section{Introduction}

The dynamics and relationships between gas inflow processes, circumnuclear star formation and the presence of an active nucleus are important, since they might provide valuable leads towards understanding the transport of gaseous material towards the nuclear regions of active galaxies. An interesting class of objects for studying these aspects are the Sérsic-Pastoriza or S-P galaxies (Sérsic \& Pastoriza 1965; Sérsic 1973) which include galaxies with diffuse and amorphous nuclei in addition to the well-known hot-spot systems. Sérsic \& Pastoriza (1967) found that galaxies with these unusual nuclear morphologies occurred in barred or mixed-type galaxies. The S-P galaxies reflect a broad spectrum of properties with some of them also harbouring an active nucleus. The existence of both starburst and an active nucleus makes them particularly interesting for studying their kinematics, evolution and possible relationships between the two forms of activity. Circumnuclear star formation has been seen in many hot-spot galaxies, and there have been suggestions of a correlation between circumnuclear rings and nuclear activity (cf. Arsenault 1989).

Theoretical studies suggest that circumnuclear rings arise due to a bar-driven inflow of gas and dust to an inner Lindblad resonance (ILR) or between two ILRs (e.g.

Send offprint requests to: D. J. Saikia,

e-mail: djs@ncra.tifr.res.in
Combes \& Gerin 1985; Athanassoula 1992; Byrd et al. 1994; Piner et al. 1995). The dense gas which accumulates in an ILR ring leads to a high star-formation rate either due to collisions of the molecular clouds (Combes \& Gerin 1985), or gravitational collapse in the ring when the density reaches a critical value (Elmegreen 1994). To understand the formation and fuelling of the AGN, the gas must flow inwards from the ILR to sub-parsec scales, and this is at present not well understood (cf. Axon \& Robinson 1996). Some simulations suggest small, steady inflow (Piner et al. 1995), while others suggest rapid inflow for brief periods (Wada \& Habe 1992; Heller \& Shlosman 1994).

In this paper we report high-resolution radio observations of the nuclear region in the barred, late-type granddesign galaxy NGC 6951, which we had earlier observed as part of a survey of $47 \mathrm{~S}-\mathrm{P}$ galaxies with the Very Large Array (VLA) at $\lambda 20$ and $6 \mathrm{~cm}$ (Saikia et al. 1994). The observations reported here are of higher resolution and better sensitivity, and clarify the radio structure of the circumnuclear region of this well-studied galaxy. New supernovae have been reported in this galaxy during 1999 and 2000 (Cao et al. 1999; Valentini et al. 2000).

\section{NGC 6951}

NGC 6951, which has been classified as a spiral galaxy of type SAB(rs)bc (de Vaucouleurs et al. 1991), has an active 

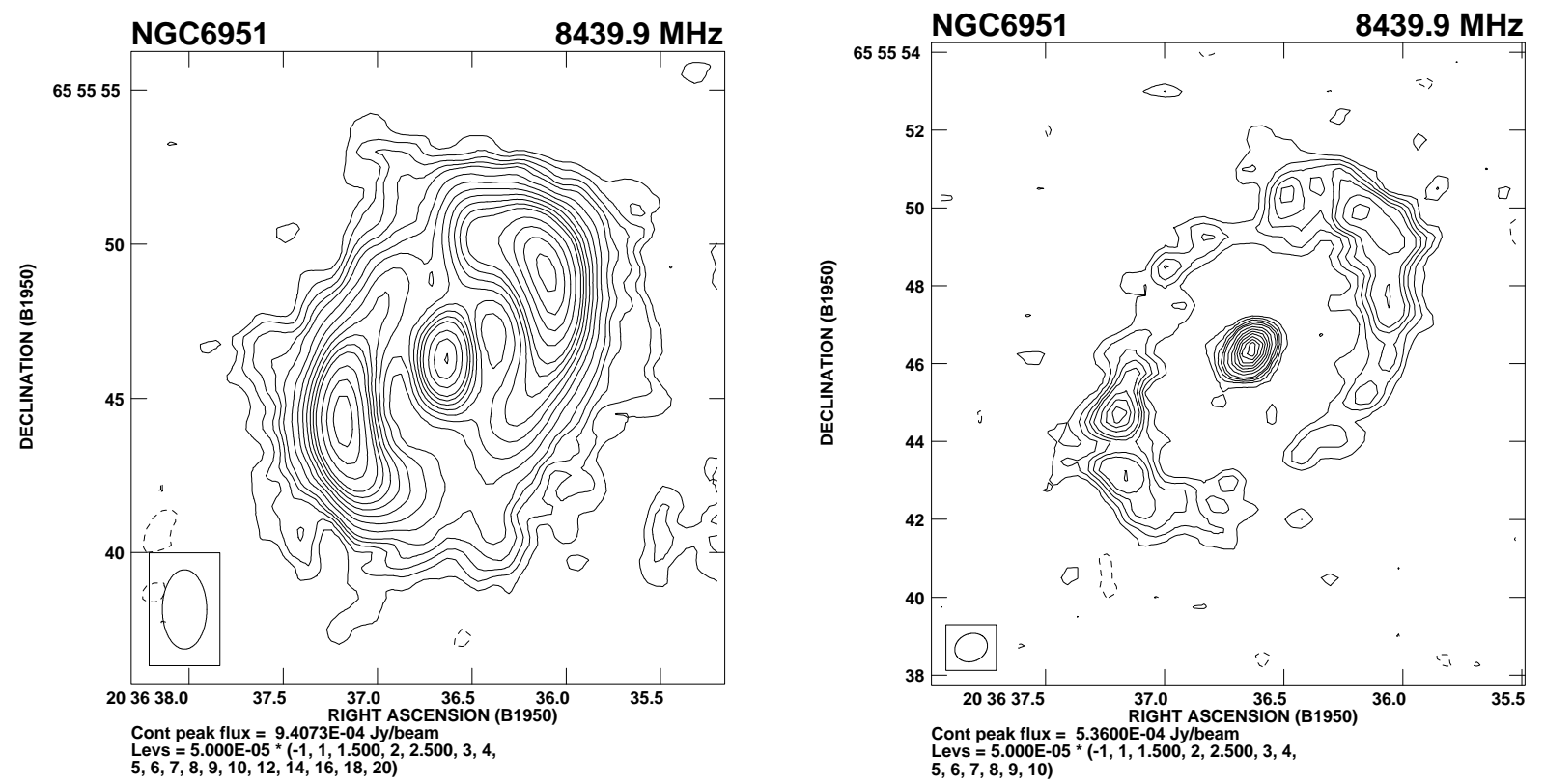

Fig. 1. Radio images of the nuclear region of NGC 6951 at $8.4 \mathrm{GHz}$ with an angular resolution of $2.57 \times 1.44$ arcsec ${ }^{2}$ along PA $0^{\circ}$ (left panel), and $0.86 \times 0.71 \operatorname{arcsec}^{2}$ along PA $113^{\circ}$ (right panel). The peak brightness and contour levels are indicated below each image.

nucleus (Boer \& Schulz 1993; Ho et al. 1995) and a circumnuclear ring of star formation (Buta \& Crocker 1993; Barth et al. 1995). The active nucleus has been classified either as a Seyfert 2 (e.g. Boer \& Schulz 1993; Ho et al. 1995, 1997a) or a LINER (Filippenko \& Sargent 1985; Muñoz-Tuñón et al. 1989; Márquez \& Moles 1993). Pérez et al. (2000) have considered the available data and have classified it as a transition object between a "very high excitation LINER and a possible nitrogen over-abundant Seyfert 2".

It is at a distance, d, of $24.1 \mathrm{Mpc}$ (Tully 1988), so that $1^{\prime \prime}$ corresponds to 117 pc. Márquez \& Moles (1993) have noted its high degree of isolation with no other galaxy within a projected distance of $1 \mathrm{Mpc}$ and $500 \mathrm{~km} \mathrm{~s}^{-1}$ in redshift, suggesting that it has been free of any gravitational influence for about $10^{9} \mathrm{yr}$.

There have been a large number of studies of this galaxy which include broad- and narrow-band observations at optical and infrared wavelengths (Buta \& Crocker 1993; Márquez \& Moles 1993; Barth et al. 1995; Wozniak et al. 1995; Elmegreen et al. 1996; Friedli et al. 1996; Knapen 1996; Rozas et al. 1996a,b; González Delgado et al. 1997; González Delgado \& Pérez 1997; Mulchaey et al. 1997; Elmegreen et al. 1999; Pérez et al. 2000), spectroscopy of the nuclear region (Filippenko \& Sargent 1985; Muñoz-Tuñón et al. 1989; Boer \& Schulz 1993; Márquez \& Moles 1993; Ho et al. 1995, 1997a,b; Pérez et al. 2000), radio images of the nuclear region (Vila et al. 1990; Saikia et al. 1994; Ho \& Ulvestad 2001, hereinafter referred to as HU) and high-resolution images of the molecular gas (Kenney et al. 1992; Kohno et al. 1999).

The most prominent features are the bar, the bulge and the spiral arms, with the bar being better defined at infrared wavelengths. The circumnuclear region has a pseudo outer, pseudo inner and circumnuclear ring, with evidence of isophotal twisting between the bar and the circumnuclear ring. The dust lanes appear as straight features from the bar edges. The ring morphology has many bright knots, with the largest concentration being towards the north-western and south-eastern sides of the ring. The hotspots or knots of emission are also seen in the infrared $K$-band and there is a general correspondence between the positions of the hotspots in $\mathrm{H} \alpha$ and in $K$ band, although in some cases there is some offset in the positions (Knapen 1996). The $\mathrm{CO}(1-0)$ emission shows a twin-peak morphology which could be due to orbit crowding near the outer ILR (Kenney et al. 1992; Kohno et al. 1999). The HCN observations by Kohno et al. show this dense gas to also have twin-peaks, but these are offset from the $\mathrm{CO}$ peaks and appear to be closely related to the radio peaks in the lower-resolution images.

\section{The nuclear radio ring in NGC 6951}

In Fig. 1 we present the convolved and full-resolution radio images of NGC 6951 at $8.4 \mathrm{GHz}$. These observations were made with the VLA B-array on 1995 October 30 and have an angular resolution of $2.57 \times 1.44 \operatorname{arcsec}^{2}$ along position angle $(\mathrm{PA}) 0^{\circ}$, and $0.86 \times 0.71 \operatorname{arcsec}^{2}$ along $\mathrm{PA} 113^{\circ}$ respectively. The rms noise level in each of the images is $\sim 0.02 \mathrm{mJy} /$ beam.

The low-resolution image shows two prominent outer peaks at RA $20^{\mathrm{h}} 36^{\mathrm{m}} 36.10$, Declination $65^{\circ} 55^{\prime} 49^{\prime \prime} \cdot 00$, and RA $20^{\mathrm{h}} 36^{\mathrm{m}} 37^{\mathrm{s}} .20$, Declination $65^{\circ} 55^{\prime} 44^{\prime \prime} .25$, with peak flux densities of 0.94 and $0.86 \mathrm{mJy} /$ beam respectively. The peak flux density of the central component is 
Table 1. The compact components.

\begin{tabular}{lccc}
\hline Source & $S_{\text {image }}$ & $S_{\text {Gauss }}$ & $\begin{array}{c}\text { Lum } \\
\times 10^{19}\end{array}$ \\
& $\mathrm{mJy} / \mathrm{b}$ & $\mathrm{mJy} / \mathrm{b}$ & $\mathrm{W} / \mathrm{Hz}$ \\
\hline $36.02+49.1$ & 0.234 & 0.23 & 1.60 \\
$36.05+46.3$ & 0.116 & 0.10 & 0.69 \\
$36.07+47.8$ & 0.205 & 0.20 & 1.39 \\
$36.16+45.2$ & 0.125 & 0.07 & 0.49 \\
$36.19+50.0$ & 0.270 & 0.24 & 1.67 \\
$36.44+43.6$ & 0.097 & 0.08 & 0.56 \\
$36.44+50.6$ & 0.157 & 0.14 & 0.97 \\
$36.64+46.3^{\mathrm{n}}$ & 0.536 & 0.54 & 3.75 \\
$36.82+49.2$ & 0.105 & 0.09 & 0.63 \\
$36.96+42.5$ & 0.109 & & $(0.76)$ \\
$36.98+48.4$ & 0.128 & 0.11 & 0.76 \\
$37.08+42.3$ & 0.145 & & $(1.01)$ \\
$37.12+45.8$ & 0.166 & & $(1.15)$ \\
$37.16+43.0$ & 0.202 & & $(1.40)$ \\
$37.20+44.7$ & 0.335 & 0.30 & 2.08 \\
\hline
\end{tabular}

$0.81 \mathrm{mJy} / \mathrm{beam}$. The high-resolution image shows the beautiful ring oriented at a position angle of $\sim 135^{\circ}$, with individual peaks of radio emission. The extent of the radio ring is $\sim 9.1 \times 6.2 \operatorname{arcsec}^{2}$, which corresponds to a linear size of $1060 \times 720 \mathrm{pc}^{2}$. The positions and flux densities of the different components were estimated by fitting Gaussians and an underlying tipped plane at the peaks of radio emission which are at least $\sim 5$ times higher than the rms noise level. These values are subject to significant uncertainties because of difficulties in defining the background flux density levels in the vicinity of each of the knots. Due to the irregular background and limited angular resolution of the observations we could not get satisfactory fits for four of the knots. All the components are listed in Table 1, along with their flux density estimates in units of mJy/beam. The components have been named such that the first four digits represent the seconds of right ascension and the digits after the + sign the arsec of declination. We have listed the peak flux densities of the components from the image (Col. 2), as well as those obtained from the Gaussian fits (Col. 3). This gives an idea of the contribution of the background at the different locations. The radio luminosities of the individual components are listed in units of $10^{19} \mathrm{~W} \mathrm{~Hz}^{-1}$ using the flux densities from the Gaussian fits, if available. The luminosities of those without satisfactory Gaussian fits have been estimated from the peak values in the image and are enclosed in parentheses. The nuclear component has been marked with the superscript " $n$ ".

The size and orientation of the ring seen at radio frequencies is roughly similar to that seen at optical, $\mathrm{H} \alpha$, infrared and $\mathrm{mm}$ wavelengths. The correspondence between the positions of the radio peaks and those seen at other wavelengths is not precise although they occur in the same general region. For example, by aligning the nuclear components, it can be seen that in the north-western region, the radio knot $36.44+50.6$ appears spatially coincident with a bright peak in the $B^{\prime}-K^{\prime}$ image (Pérez et al. 2000) as well as a prominent peak in the $\mathrm{H} \alpha$ contours (González Delgado et al. 1997). On the other hand, there are no such corresponding bright features for the nearby brighter radio components $36.19+50.0$ and $36.02+49.1$.

The separation between the successive peaks of radio emission listed in Table 1 ranges from $\sim 0$ ". 8 to $33^{\prime \prime} 5$. These separations are reasonably uniformly distributed between $0 . \prime 8$ and $2 . .6$, with only the largest separation being beyond this range. There does not appear to be a regular period in the separation of the peaks of emission. If the radio peaks trace the star forming regions, one would expect these to be regularly spaced in the gravitational instability models (Elmegreen 1994).

The two most prominent regions of radio emission are towards the north-west and the south-east. From $\mathrm{CO}(1-0)$ and $\mathrm{HCN}(1-0)$ observations, Kohno et al. (1999) find that although both $\mathrm{CO}$ and HCN emission are dominated by "twin-peaks" morphology, the HCN emission is better correlated spatially with the peaks of radio emission, which are identified with the regions of massive star formation. In this case, the peaks of $\mathrm{CO}$ emission are identified with the region of crowding of the $x_{1}$ and $x_{2}$ orbits.

\subsection{Nature of radio emission}

The radio emission from the circumnuclear region is possibly a mixture of thermal and non-thermal emission with the discrete components being either supernova remnants (SNRs) or H II regions. The diagnostics which are usually employed to distinguish them are the brightness temperatures, as in the starburst galaxy Arp 220 (Smith et al. 1998), and radio spectral indices. The radio components are usually too weak for polarization measurements.

The brightness temperature of the components in NGC 6951 is small, $\lesssim 15 \mathrm{~K}$, and does not provide an unambiguous resolution of the nature of the compact components. Spectral index information on the individual knots is not available; however the spectral index, $\alpha$ defined as $S \propto \nu^{-\alpha}$, of the entire circumnuclear emission estimated from scaled-array VLA observations between $\lambda 20$ and $6 \mathrm{~cm}$ is $0.84(\mathrm{HU})$. Convolving the $\lambda 3.6 \mathrm{~cm}$ image to lower resolutions and comparing with the images of Saikia et al. (1994) and HU also suggests a non-thermal spectrum.

However, a few caveats need to be borne in mind while examining the nature of the emission of the components based on their spectra. With inadequate resolution H II regions and SNRs may not be resolved from each other (cf. Gordon et al. 1999). Also, although the overall spectrum from coarser resolution might appear non-thermal, individual compact components identified from higherresolution observations may be due to $\mathrm{H}$ II regions. An 
example of this is the blue compact galaxy Henize 2-10 which is an archetypal starburst galaxy containing a large population of Wolf-Rayet stars (Conti 1991). The integrated radio spectrum estimated from observations largely with an angular resolution of about a kpc has a nonthermal spectrum, with $\alpha \sim 0.54$, while the compact radio knots observed with a resolution of $\sim 30$ pc have a flat or inverted spectrum (Kobulnicky \& Johnson 1999). These are identified with dense, compact H II regions which are strong sources of free-free emission. For example, the features in Henize $2-10(d \sim 9 \mathrm{Mpc})$ have a luminosity of $\sim 9 \times 10^{18} \mathrm{~W} \mathrm{~Hz}^{-1}$ at $\lambda 3.6 \mathrm{~cm}$, electron densities of up to $5000 \mathrm{~cm}^{-3}$ and harbour the youngest "super-star clusters" (Kobulnicky \& Johnson 1999). A similar feature has been reported in the nuclear region of NGC 5253 (Turner et al. $1998,2000)$ which has a luminosity of $\sim 2 \times 10^{19} \mathrm{~W} \mathrm{~Hz}^{-1}$ at $\lambda 2 \mathrm{~cm}$. Infrared observations confirm it to be an $\mathrm{H}$ II region excited by a dense cluster of young stars (Gorjian et al. 2001).

The nature of the compact radio components in other galaxies with either a circumnuclear ring or a nuclear starburst might provide some clues towards understanding the nature of the components in NGC 6951. A number of these galaxies have been observed at more than one frequency. Amongst the galaxies with radio rings, the radio spectrum of the ring in NGC 613 was found to be $\sim 0.65 \pm 0.10$ (Hummel \& Jörsäter 1992), the components in NGC 1097 (Hummel et al. 1987) and NGC 1365 (Saikia et al. 1994; Sandqvist et al. 1995; Stevens et al. 1999) tend to have non-thermal spectra, as well as the ring of emission in the Seyfert galaxy NGC 7469 (Wilson et al. 1991). In NGC 4736, which has a continuum ring, Duric \& Dittmar (1988) found 9 of their compact components to have a thermal spectrum between $\lambda 20$ and $6 \mathrm{~cm}$, and the remaining 10 to have a non-thermal spectrum. Turner \& Ho (1994) observed this galaxy at $\lambda 6$ and $2 \mathrm{~cm}$, and found 9 of their 14 sources to have $\alpha<0.2$.

The compact components in these systems are a mixture of both SNRs and H II regions occuring in an area of high star formation. The proportion of these two types of objects has been investigated in some detail in the archetypal starburst galaxies M 82 (Unger et al. 1984; Kronberg et al. 1985) and NGC 253 (Antonucci \& Ulvestad 1988). Spectral studies of selected components in M 82 (Wills et al. 1997; Allen \& Kronberg 1998) showed that of the 26 components, only one was identified with a thermal emission spectrum. However, more recent observations of a further 20 largely weaker components suggest that only 5 of these are SNRs while the remaining 15 are likely to be H II regions (McDonald 2001). Thus about two-thirds of the compact objects are identified with SNRs. In NGC 253 about a half of the sources with spectral information have flat spectra and are dominated by emission from $\mathrm{H}$ II regions, while the remaining half have steep spectra and are identified with SNRs (Ulvestad \& Antonucci 1991, 1997). A similar situation is also seen in the nearby merger NGC 4038/4039 (the Antennae), where about a third of the objects have been suggested to be thermal and the remaining two-thirds non-thermal (Neff \& Ulvestad 2000). The spectral indices of the components in NGC 1808 tend to be nonthermal (Saikia et al. 1990; Collison et al. 1994). In the starburst galaxy NGC 2146 which has an $S$-shaped structure with several knots of emission (Kronberg \& Biermann 1981; Saikia et al. 1994), spectral index measurements suggest three sources to be good candidates for SNRs or radio supernovae, and 6 to be associated with compact, dense H II regions (Tarchi et al. 2000). Based on these studies of a wide variety of starburst galaxies, it is reasonable to assume that the ring in NGC 6951 consists of a mixture of both these types of objects, although our linear resolution of about $100 \times 83 \mathrm{pc}^{2}$ is inadequate to identify individual SNRs and HII regions.

\subsection{Radio luminosity and supernova rate}

The compact components seen in NGC 6951 have a median luminosity of $10^{19} \mathrm{~W} \mathrm{~Hz}^{-1}$ at $\lambda 3.6 \mathrm{~cm}$. The value is similar when one considers those with Gaussian fits as well as for the entire list. The luminosity distribution is skewed towards lower values, with a tail extending towards higher luminosities, suggesting that we have not been able to identify the weaker components from the existing observations.

It is interesting to compare the values obtained for NGC 6951 with those obtained for other galaxies exhibiting high star formation rates. For ease of comparison we have considered those for which observations have been made at $\lambda 3.6 \mathrm{~cm}$. The median luminosity of the components in NGC $253(d \sim 2.5 \mathrm{Mpc})$ observed with a linear resolution of $\sim 3 \mathrm{pc}$ is $\sim 4.5 \times 10^{17} \mathrm{~W} \mathrm{~Hz}^{-1}$ (Ulvestad \& Antonucci 1991), while those in M82 ( $d \sim 3.4 \mathrm{Mpc}$ ) observed with a similar linear resolution has a median value of $\sim 2 \times 10^{18} \mathrm{~W} \mathrm{~Hz}^{-1}$ (Huang et al. 1994; Allen \& Kronberg 1998). Considering more distant galaxies, the median luminosity for the archetypal starburst galaxy in the southern hemisphere, NGC 1808 ( $d \sim 10.9 \mathrm{Mpc}$ ), is about $8 \times 10^{18} \mathrm{~W} \mathrm{~Hz}^{-1}$ with a linear resolution of $30 \mathrm{pc}$ (Collison et al. 1994), while the knots in NGC $1365(d \sim$ $20 \mathrm{Mpc}$ ) has a median luminosity of $\sim 3.5 \times 10^{19} \mathrm{~W} \mathrm{~Hz}^{-1}$ with a linear resolution of about a 100 pc (Stevens et al. 1999). The increase in luminosity for the more distant systems is largely due to the coarser linear resolution of the observations. To examine this we have convolved a multiconfiguration VLA image of M 82 with a linear resolution of $\sim 6 \mathrm{pc}$ to a resolution comparable with our observations of NGC 6951. In the convolved image, the individual components are indistinguishable and the peak brightness has increased by a factor of $\sim 16$, corresponding to a luminosity of $6.1 \times 10^{20} \mathrm{~W} \mathrm{~Hz}^{-1}$ at $5 \mathrm{GHz}$. This is more luminous than the individual radio components in NGC 6951. A more detailed comparison would require observations of NGC 6951 with higher linear resolution.

We estimate the $S N$ rate in NGC 6951 using the model of Condon \& Yin (1990) which was based on data for our Galaxy. Assuming that the entire emission at $1465 \mathrm{MHz}$ 
(Hummel et al. 1985) is non-thermal, we estimate the $S N$ rate to be $\sim 0.07 \mathrm{yr}^{-1}$. Bearing in mind that the emission at $1465 \mathrm{MHz}$ could also contain a thermal contribution, the $S N$ rate in NGC 6951 appears to be reasonably consistent with estimates of $\sim 0.1 \mathrm{yr}^{-1}$ for M 82 (e.g. Huang et al. 1994), $\lesssim 0.1$ to 0.3 for NGC 253 (Ulvestad \& Antonucci 1997), $\sim 0.1 \mathrm{yr}^{-1}$ for the clumpy irregular starburst galaxy Mrk 325 (Condon \& Yin 1990) and also for the starburst galaxy NGC 3448 of the Arp 205 system (Noreau \& Kronberg 1987).

\subsection{The central component}

By fitting a two-dimensional Gaussian and an underlying tipped plane to the central component, we find it to be resolved with a deconvolved angular size of $0.7 \times 0.2 \operatorname{arcsec}^{2}$ along a PA of $156^{\circ}$. This corresponds to a linear dimension of $\sim 80 \times 20 \mathrm{pc}^{2}$. HU also find evidence of a similar extension but are unable to confirm it because of the coarser resolution of their observations with the restoring beam being elongated along a similar PA. The $\mathrm{H} \alpha$ image of Knapen (1996) also seems to show a similar extension. The spectral index of the nuclear component obtained by smoothing the $\lambda 3.6 \mathrm{~cm}$ image to that of the lower-resolution $\lambda 6 \mathrm{~cm}$ image of Saikia et al. (1994) is $\sim 0.6$. The scaled-array observations of HU yield slightly higher values of 0.69 and 0.90 between $\lambda 20$ and $6 \mathrm{~cm}$ for the peak and integrated flux densities respectively. The supernova rate estimated from the nuclear radio flux density (cf. Colina \& Pérez-Olea 1992 ) is about $0.003 \mathrm{yr}^{-1}$, which is much smaller than the $S N$ rate in bright nuclear starbursts. As pointed out by Pérez et al. (2000), the low $S N$ rate is consistent with the faintness of the nucleus in $\mathrm{H} \alpha$. The resolved central component, which is elongated and has a steep spectral index, could be due to a small-scale, weak nuclear jet from the Seyfert/LINER nucleus.

\section{Conclusions}

High-resolution radio observations of the circumnuclear region of NGC 6951 reveal a ring of radio emission with many discrete components and a slightly resolved nuclear component. The discrete components have a median luminosity of $10^{19} \mathrm{~W} \mathrm{~Hz}^{-1}$ when observed with a linear resolution of $\sim 90 \mathrm{pc}$, and are likely to consist of a mixture of both SNRs and HiI regions. The $S N$ rate has been estimated to be $\sim 0.07 \mathrm{yr}^{-1}$, which is reasonably consistent with those found in some of the archetypal starburst galaxies. The central component, which is resolved and has a steep radio spectrum, could be due to a small-scale jet from the active nucleus.

Acknowledgements. The National Radio Astronomy Observatory is a facility of the National Science Foundation operated under co-operative agreement by Associated Universities, Inc. We have made use of the NASA/IPAC Extragalactic Database (NED), which is operated by the Jet Propulsion Laboratory, California Institute of Technology under contract with the National Aeronautics and Space
Administration. We thank Jim Ulvestad for several comments and suggestions which have improved the paper significantly. We also thank Graham Smith and Peter Thomasson for their detailed comments on the manuscript. One of us (DJS) would like to thank the Cave Lecture Fund and the Principal's Development Fund of Queen's University, Kingston, and Director, Jodrell Bank Observatory for financial support, and Judith Irwin, Dieter Brueckner and Peter Thomasson for hospitality during different phases of this piece of work.

\section{References}

Allen, M. L., \& Kronberg, P. P. 1998, ApJ, 502, 218

Antonucci, R. R. J., \& Ulvestad, J. S. 1988, ApJ, 330, L97

Arsenault, R. 1989, A\&A, 217, 66

Athanassoula, E. 1992, MNRAS, 259, 345

Axon, D. J., \& Robinson, A. 1996, in Barred galaxies and circumnuclear activity, ed. Aa Sandqvist, \& P. O. Lindblad (Springer), 223

Barth, A. J., Ho, L. C., Filippenko, A. V., \& Sargent, W. L. 1995, AJ, 110, 1009

Boer, B., \& Schulz, H. 1993, A\&A, 277, 397

Buta, R., \& Crocker, D. A. 1993, AJ, 105, 1344

Byrd, G., Rautianen, P., Salo, H., Buta, R., \& Crocker, D. A. 1994, AJ, 108, 476

Cao, L., Qiu, Y. L., Qiao, Q. Y., \& Hu, J. Y. 1999, IAUC, 7288

Colina, L., \& Pérez-Olea, D. 1992, MNRAS, 259, 709

Collison, P. M., Saikia, D. J., Pedlar, A., Axon, D. J., \& Unger, S. W. 1994, MNRAS, 268, 203

Combes, F., \& Gerin, M. 1985, A\&A, 150, 327

Condon, J. J., \& Yin, Q. F. 1990, ApJ, 357, 97

Conti, P. S. 1991, ApJ, 377115

de Vaucouleurs, G., de Vaucouleurs, A., Corwin, H. G., et al. 1991, Third Reference Catalogue of Bright Galaxies (Springer, New York)

Duric, N., \& Dittmar, M. R. 1988, ApJ, 332, L67

Elmegreen, G. B. 1994, ApJ, 425, L73

Elmegreen, D. M., Elmegreen, B. G., Chromey, F. R., et al. 1996, AJ, 111, 1880

Elmegreen, D. M., Chromey, F. R., Sawyer, J. E., \& Reinfeld, E. L. 1999, AJ, 118, 777

Filippenko, A. V., \& Sargent, W. L. W. 1985, ApJS, 57, 503

Friedli, D., Wozniak, H., Rieke, M., et al. 1996, A\&AS, 118, 461

González Delgado, R. M., \& Pérez, E. 1997, ApJS, 108, 199

González Delgado, R. M., Pérez, E., Tadhunter, C. N., et al. 1997, ApJS, 108, 155

Gordon, S. M., Duric, N., Kirshner, R. P., Goss, W. M., \& Viallefond, F., 1999, ApJS, 120, 247

Gorjian, V., Turner, J. L., \& Beck, S. C., ApJ, 554, L29

Heller, C. H., \& Shlosman, I. 1994, ApJ, 424, 84

Ho, L. C., Filippenko, A. V., \& Sargent, W. L. W. 1995, ApJS, 98, 477

Ho, L. C., Filippenko, A. V., \& Sargent, W. L. W. 1997a, ApJS, 112, 315

Ho, L. C., Filippenko, A. V., Sargent, W. L. W., et al. 1997b, ApJS, 112, 391

Ho, L. C., \& Ulvestad, J. S. 2001, ApJS, 133, 77 (HU)

Huang, Z. P., Thuan, T. X., Chevalier, R. A., Condon, J. J., \& Yin, Q. F. 1994, ApJ, 424, 114

Hummel, E., \& Jörsäter, S. 1992, A\&A, 261, 85

Hummel, E., Pedlar, A., van der Hulst, J. M., \& Davies, R. D. 1985, A\&AS, 60, 293 
Hummel, E., van der Hulst, J. M., \& Keel, W. C. 1987, A\&A, 172,32

Kenney, J. D. P., Wilson, C. D., Scoville, N. Z., et al. 1992, ApJ, 395, L79

Knapen, J. H. 1996, in ed. Aa. Sandqvist, \& P. O. Lindblad, Barred galaxies and circumnuclear activity (Springer), 233

Kobulnicky, H. A., \& Johnson, K. E. 1999, ApJ, 527, 154

Kohno, K., Kawabe, R., \& Vila-Vilaró, B. 1999, ApJ, 511, 157

Kronberg, P. P., \& Biermann, P. 1981, ApJ, 243, 89

Kronberg, P. P., Biermann, P., \& Schwab, F. R. 1985, ApJ, 291,693

Márquez, I., \& Moles, M. 1993, AJ, 105, 2090

McDonald, A. M. 2001, Ph.D. Thesis, University of Manchester

Mulchaey, J. S., Regan, M. W., \& Kundu, A. 1997, ApJS, 110, 299

Muñoz-Tuñón, C., Vilchez, J. M., Castañeda, H., et al. 1989, Ap\&SS, 157, 165

Neff, S. G., \& Ulvestad, J. S. 2000, AJ, 120, 670

Noreau, L., \& Kronberg, P. P. 1987, AJ, 93, 1045

Pérez, E., Márquez, I., Marrero, I., et al. 2000, A\&A, 353, 893

Piner, B. G., Stone, J. M., \& Teuben, P. J. 1995, ApJ, 449, 508

Rozas, M., Beckman, J. E., \& Knapen, J. H. 1996a, A\&A, 307, 735

Rozas, M., Beckman, J. E., \& Knapen, J. H. 1996b, A\&A, 312, 275

Saikia, D. J., Unger, S. W., Pedlar, A., et al. 1990, MNRAS, 245,397

Saikia, D. J., Pedlar, A., Unger, S. W., \& Axon, D. J. 1994, MNRAS, 270, 46
Sandqvist, Aa., Jörsäter, S., \& Lindblad, P. O. 1995, A\&A, 295,585

Sérsic, J. L. 1973, PASP, 85, 103

Sérsic, J. L., \& Pastoriza, M. 1965, PASP, 77, 287

Sérsic, J. L., \& Pastoriza, M. 1967, PASP, 79, 152

Smith, H. E., Lonsdale, C. J., Lonsdale, C. J., \& Diamond, P. J. 1998, ApJ, 493, L17

Stevens, I. R., Forbes, D. A., \& Norris, R. P. 1999, MNRAS, 306,479

Tarchi, A., Neininger, N., Greve, A., et al., A\&A, 358, 95

Tully, R. B. 1988, Nearby Galaxies Catalogue (Cambridge University Press, Cambridge)

Turner, J. L., \& Ho, P. T. P. 1994, ApJ, 421, 122

Turner, J. L., Ho, P. T. P., \& Beck, S. C. 1998, AJ, 116, 1212

Turner, J. L., Beck, S. C., \& Ho, P. T. P. 2000, ApJ, 532, L109

Ulvestad, J. S., \& Antonucci, R. R. J. 1991, AJ, 102, 875

Ulvestad, J. S., \& Antonucci, R. R. J. 1997, ApJ, 488, 621

Unger, S. W., Pedlar, A., Axon, D. J., Wilkinson, P. N., \& Appleton, P. N. 1984, MNRAS, 211, 783

Valentini, G., Di Carlo, E., \& Guidubaldi, D. 2000, IAUC, 7351

Vila, M. B., Pedlar, A., Davies, R. D., et al. 1990, MNRAS, 242, 379

Wada, K., \& Habe, A. 1992, MNRAS, 258, 82

Wills, K. A., Pedlar, A., Muxlow, T. W. B., \& Wilkinson, P. N. 1997, MNRAS, 291, 517

Wilson, A. S., Helfer, T. T., Haniff, C. A., \& Ward, M. J. 1991, ApJ, 381, 79

Wozniak, H., Friedli, D., Martinet, L., et al. 1995, A\&AS, 111, 115 